\title{
PROJETO DE REABILITAÇÃO CARDIOVASCULAR NO RITMO DO SAMBA DE UMA UNIVERSIDADE PÚBLICA DO ESTADO DE SÃO PAULO
}

Thamyres Rangel Mendes Barros, Juliana dos Santos Proença, Charles Domhof Maeda, Ana Carolina Mariano Inacio, Bruna Izadora Spinardi Alves, Natacha de Lima Gervazoni, Jéssica Mariany Rodrigues da Silva, Viviane Akemi Kakazu, Beatriz Espanhol Garcia, Paula Rapchan dos Santos Torquato, Felipe Ribeiro, Roselene Modolo Regueiro Lorençoni.

Universidade Estadual Paulista - UNESP - UNESP, Departamento de Fisioterapia, Presidente Prudente, SP. E-mail: tamifani@hotmail.com. Pró-reitora de Extensão Universitária - PROEX.

\section{RESUMO}

O objetivo foi Descrever as atividades desenvolvidas e caracterizar os participantes do Projeto de Reabilitação Cardiovascular no Ritmo do Samba. Estudo descritivo transversal, realizado com 13 mulheres que participam de um grupo de reabilitação por meio do samba. Dados pessoais, medicamentos, fatores de risco (FR) e mensuração de frequência cardíaca ( $F C$ ), pressão arterial (PA) e medidas antropométricas foram feitas para caracterização. A PA sistólica e diastólica foram de $121,53 \pm 20,75 \mathrm{mmHg}$ e $80,76 \pm 12,55 \mathrm{mmHg}$, respectivamente. A FC de repouso de $75,61 \pm 9,72$ bpm, circunferência de cintura de $96,42 \pm 9,34 \mathrm{~cm}$, circunferência de quadril de $109,42 \pm 8,15 \mathrm{~cm}$ e a relação entre eles de $0,87 \pm 0,05 \mathrm{~cm}$. Histórico familiar $(N=10)$, sedentarismo $(N=8)$, obesidade ( $N$ $=7)$ e estresse $(N=7)$ foram os FR mais prevalentes. Conclui-se que a população participante do projeto é exclusivamente feminina, com idade acima de 50 anos e PA dentro da normalidade. Ainda, os principais FR foram o histórico familiar, sedentarismo, obesidade e estresse.

Palavras-chave: Reabilitação cardíaca; Fatores de Risco; Dança.

\section{CARDIOVASCULAR REHABILITATION PROJECT IN THE SAMBA RHYTHM OF THE PUBLIC UNIVERSITY OF SÃO PAULO STATE}

\begin{abstract}
The objective was to describe the activities developed and characterize the participants of the Cardiovascular Rehabilitation Project in the Samba Rhythm. A descriptive cross-sectional study was carried out with 13 women participating in a rehabilitation group through samba. Personal data, medications, risk factors (RF) and heart rate (HR) measurements, blood pressure (BP) and anthropometric measurements were made for characterization. The systolic and diastolic BP were $121.53 \pm 20.75 \mathrm{mmHg}$ and $80.76 \pm 12.55 \mathrm{mmHg}$, respectively. The resting HR of $75.61 \pm 9.72 \mathrm{bpm}$, waist circumference of $96.42 \pm 9.34 \mathrm{~cm}$, hip circumference of $109.42 \pm 8.15 \mathrm{~cm}$ and the relation between them of $0.87 \pm 0,05 \mathrm{~cm}$. Family history $(N=10)$, sedentary lifestyle $(N=8)$, obesity $(N=$ 7) and stress $(\mathrm{N}=7)$ were the most prevalent RF. It has concluded that the majority of the project's participants is exclusively female, with an age over 50 years old and BP within normality. Also, the main RF were the family history, sedentary lifestyle, obesity and stress.
\end{abstract}

Keywords: Cardiac Rehabilitation; Risk Factors; Dancing. 


\section{INTRODUÇÃO}

As doenças cardiovasculares (DCV) são responsáveis pela morte de aproximadamente 15 milhões de pessoas a cada ano ${ }^{1}$ e sua incidência aumentou em aproximadamente um terço entre 1990 e $2010^{2}$. Ainda, as DCV podem promover diversas condições negativas para o organismo, como por exemplo incapacidade física e invalidez, além de contribuir significativamente para o aumento nas despesas com saúde e problemas para a sociedade em geral ${ }^{3}$.

Os fatores de risco (FR) como a obesidade, tabagismo, diabetes mellitus (DM) e hipertensão arterial (HA) estão diretamente associados ao surgimento de DCV, e a prevalência de ambos tem seguido um aumento de forma paralela, reforçando assim a estreita relação entre as DCV e o estilo de vida do indivíduo ${ }^{4}$.

Dessa forma, medidas que promovam uma melhora na qualidade de vida dos indivíduos, bem como mudança nos hábitos de vida diário, se tornam essenciais tanto para o controle quanto para o tratamento de indivíduos com FR ou DCV, podendo destacar assim, os programas de Reabilitação Cardiovascular.

A relevância dos programas de Reabilitação Cardiovascular, quando associada a mudanças no estilo de vida, promove uma diminuição da mortalidade e redução dos $F R$, sobretudo para os indivíduos classificados como de alto risco de desenvolver DCV. Uma vez que os programas de Reabilitação Cardiovascular são compostos pela prática de atividade física, é capaz de gerar uma melhora da capacidade aeróbia, bem como uma redução dos sintomas relacionados ao esforço e o aperfeiçoamento dos sistemas respiratório, cardiovascular e musculoesquelético ${ }^{4}$.

Apesar dos benefícios conhecidos que os programas de Reabilitação Cardiovascular trazem para os pacientes com FR ou DCV, existe uma dificuldade na adesão ao tratamento e motivação dos pacientes que impossibilita obter melhores resultado ${ }^{5,6}$. Neste contexto, os profissionais se sentem motivados e encorajados a buscarem novas alternativas de programas para controle de FR e tratamento para essas doenças ${ }^{7,8}$.

Neste contexto, os programas de Reabilitação Cardiovascular que utilizam danças, têm mostrado efeitos favoráveis no aprimoramento da aptidão física e das condições cardiovasculares semelhante ao encontrado em programas tradicionais de treinamento físico ${ }^{8,9}$

Pensando nisso, a implementação de novas modalidades de tratamento que visam promover a melhora da qualidade de vida em indivíduos que apresentem FR para DCV, bem como, auxiliem a sua permanência no mesmo, devem ser incentivadas. Portanto, esse trabalho tem por objetivo descrever as atividades desenvolvidas no Projeto de Reabilitação Cardiovascular no Ritmo do Samba no Centro de Estudos e Atendimento em Fisioterapia e Reabilitação da FCT/UNESP e caracterizar os indivíduos que participam atualmente.

\section{MATERIAIS E MÉTODOS}

Trata-se de um estudo descritivo transversal realizado com as integrantes do programa de extensão universitária de reabilitação cardiovascular utilizando o ritmo do samba. Como critério de inclusão, consideramos que os indivíduos deveriam apresentar idade superior a 18 anos, ter ou não vínculo empregatício com a universidade e apresentar um ou mais fatores de risco para doenças cardiovasculares. Como critério de exclusão, os indivíduos não poderiam apresentar DCV.

Todos os voluntários foram devidamente informados sobre os procedimentos e objetivos deste estudo, e após concordarem, assinaram um termo de consentimento, passando a fazer parte efetivamente do mesmo. Os procedimentos do estudo foram aprovados pelo Comitê de Ética em Pesquisa da FCT/UNESP com o número CEP: 504292115.70000.5402.

Inicialmente, foi realizada uma entrevista com os participantes para obter informações a respeito de dados pessoais, medicamentos utilizados, fatores de risco e mensuração da frequência cardíaca (FC), pressão arterial (PA) e medidas antropométricas. 
Os fatores de risco analisados, seguiram as recomendações para a classificação de risco cardíaco segundo a $\mathrm{ACSM}^{10}$, sendo estes: obesidade, estresse, sedentarismo, hipertensão arterial, tabagismo, diabetes, dislipidemia, histórico familiar e alcoolismo.

Para a verificação da frequência cardíaca (FC) foi realizada a palpação da artéria radial ou braquial durante 1 minuto. Ainda, foi realizada a mensuração da pressão arterial de forma indireta, seguindo as recomendações da 7ạ Diretriz Brasileira de Hipertensão Arterial, por meio de um esfigmomanômetro aneroide (Welch Allyn - Tycos, New York, USA) e um estetoscópio (Littmann, Saint Paul, USA $)^{11}$.

Para o cálculo do índice de massa corporal (IMC) foi realizada a mensuração do peso corporal por meio de uma balança digital eletrônica (Plenna, Lumina mea 02550, Brasil) e sua estatura realizada por meio de um estadiômetro (Sanny, Personal Caprice, Brasil). Após a obtenção dos dados, o IMC foi calculado pela seguinte equação: IMC = peso/altura ${ }^{2}\left(\mathrm{~kg} / \mathrm{m}^{2}\right)^{12}$.

Para a mensuração da circunferência de cintura (CC), foi realizada com uma fita métrica e teve como referência a menor circunferência entre as costelas e a crista ilíaca. A circunferência de quadril (CQ) foi mensurada a partir da maior protuberância glútea. A partir dessas medidas foi realizado o cálculo da relação cintura/quadril (RCQ).

Em relação ao protocolo de reabilitação cardiovascular no ritmo do samba, duas sessões são realizadas semanalmente e tem duração de 60 minutos. São aferidos os parâmetros de FC e PA no início e no final e é divido em 3 partes: aquecimento, resistência e relaxamento.

O aquecimento tem duração de 15 minutos e tem por objetivo de adaptar o sistema cardiovascular, onde os indivíduos realizam coreografias com músicas previamente selecionadas e classificadas em um ritmo lento. A fase de resistência compreende 35 minutos, e são realizadas coreografias com um ritmo moderado e rápido em busca de alcançar a reserva de frequência cardíaca (RFC). Por fim o relaxamento dura 10 minutos para redução da FC, onde nos 5 primeiros são utilizadas músicas mais lentas e nos outros 5 eles ficam deitados em silêncio.

Para análise dos dados foi utilizado estatística descritiva e os resultados foram apresentados em média, desvio-padrões, números absolutos e percentuais.

\section{RESULTADOS}

A Tabela 1 mostra os valores médios e seus respectivos desvios-padrão das variáveis idade, peso, altura, IMC e valores em percentual da classificação de gênero para a caracterização da população avaliada.

Tabela 1. Caracterização dos indivíduos que participam do programa de reabilitação cardiovascular no ritmo do samba.

\begin{tabular}{cc}
\hline Variáveis & População (13) \\
\hline Idade (anos) & $52,52 \pm 8,96$ \\
Peso (Kg) & $79,11 \pm 12,35$ \\
Altura (cm) & $160,61 \pm 6,50$ \\
IMC (kg/m2) & $30,69 \pm 5,21$ \\
Gênero Feminino & $13(100 \%)$
\end{tabular}

Legenda: \pm = média \pm desvio padrão; $\mathrm{Kg}=$ quilogramas; $\mathrm{cm}=$ centímetros; $\mathrm{m}=$ metros; $\mathrm{m} 2$ = metros quadrados

Em relação aos parâmetros cardiovasculares as participantes apresentaram PAS média de $121,53 \pm 20,75 \mathrm{mmHg}$, PAD média de 80,76 \pm 12,55 e FC de 75,61 \pm 9,72 batimentos por minuto.

Considerando os valores de circunferências, as participantes apresentaram valores médios de $\mathrm{CC}$ de $96,42 \pm 9,34 \mathrm{~cm}, \mathrm{CQ}$ de 109,42 $\pm 8,15 \mathrm{~cm}$ e RCQ de 0,87 $\pm 0,05 \mathrm{~cm}$. 
Já a tabela 2 mostra a prevalência de fatores de risco nessa população em ordem decrescente, indicando maior incidência de FR relacionados ao histórico familiar $(N=10)$, sedentarismo $(N=8)$, obesidade $(N=7)$ e estresse $(N=7)$.

Tabela 2. Incidência dos fatores de risco cardiovasculares nas participantes do programa de reabilitação no ritmo do samba.

\begin{tabular}{cc}
\hline Variáveis & População (13) \\
\hline Histórico Familiar & $76,92 \%$ \\
Sedentarismo & $61,53 \%$ \\
Obesidade & $53,84 \%$ \\
Estresse & $53,84 \%$ \\
Hipertensão Arterial & $38,46 \%$ \\
Dislipidemia & $15,38 \%$ \\
Diabetes & $7,69 \%$ \\
Tabagismo & $0 \%$ \\
Alcoolismo & $0 \%$
\end{tabular}

Legenda: \% = valores expressos em números percentuais.

\section{DISCUSSÃO}

Por meio deste estudo pode-se observar que os participantes do programa de reabilitação no ritmo do samba são compostos exclusivamente por mulheres de meia idade, com maior incidência de FR relacionados ao histórico familiar, sedentarismo, obesidade e estresse.

Em relação a composição corporal, as participantes em questão apresentaram IMC > de 25 , e segundo a Associação Brasileira de Estudo Sobre a Obesidade (ABESO) ${ }^{12}$ indivíduos que apresentam estes valores de IMC estão mais suscetíveis a desenvolver DCV e o risco torna-se ainda maior quando associados aos fatores de risco que as mesmas apresentam.

Ainda, a população participante do programa de reabilitação é exclusivamente feminina, o que corrobora com dados fornecidos pelo DataSUS ${ }^{13}$, onde indicam que as mulheres cuidam mais de sua saúde e por isso estão mais presentes em assuntos relacionados à saúde.

As praticantes apresentaram valores médios para PAS e PAD dentro dos considerados normais, uma vez que isso pode ser justificado pelo fato de que $38,46 \%(N=5)$ das pacientes realizam tratamento medicamentoso para controle, além de estarem inseridas em um programa que utiliza o exercício físico como principal ferramenta, o que auxilia também no controle dos valores de PA.

Em relação aos valores de RCQ, foi possível observar que a maior parte das integrantes apresentaram valores médios acima de $0,85 \mathrm{~cm}$. Estes valores segundo Moraes et al $^{14}$, predispõe o indivíduo a um risco ainda mais elevado de desenvolver DCV.

Quanto a incidência de fatores de risco, notamos que o mais ocorrente foi o histórico familiar, este quando associado principalmente parentes de 10 grau que apresentaram casos de morte súbita e infarto prematuro e fatores de risco como diabetes, HAS e dislipidemia isso eleva o risco para desenvolvimento de doença coronariana, possuem um risco ainda maior para o desenvolvimento de $\mathrm{DCV}^{15}$. Além da predisposição genética, os antecedentes familiares estão relacionados a hábitos inadequados no estilo de vida familiar, justificando assim a importância da inserção de medidas preventivas e educacionais a respeito de como modificar os hábitos de vida.

Nesse sentido é indispensável a busca por programas que englobem a prevenção, para controle dos diversos fatores de risco, reduzindo assim o risco de desenvolver doenças cardiovasculares. Dessa forma, programas de prevenção que englobam a dança têm recebido crescente interesse devido aos benefícios que a mesma traz e a forma que ela auxilia os participantes a permanecerem no programa por ser uma atividade física prazerosa. 


\section{CONCLUSÃO}

Conclui-se que a maioria da população participante do projeto é exclusivamente feminina e possuem idade acima de 50 anos. Ainda, apresentam valores médios de PA dentro da normalidade, bem como o histórico familiar, sedentarismo, obesidade e estresse como os principais fatores de risco cardiovasculares associados.

\section{REFERÊNCIAS}

1. Simão $M$, Hayashida $M$, Santos $C B$ et al. Hypertension among undergraduate students from Lubango. Angola. Rev Lat Am Enfermagem. 2008;16:672-78. https://doi.org/10.1590/S010411692008000400004.

2. Lozano R, Naghavi M, Foreman K, et al. Global and regional mortality from 235 causes of death for 20 age groups in 1990 and 2010: a systematic analysis for the Global Burden of Disease Study. The Lancet. 2012;380:2095-128. http://dx.doi.org/10.1016/S0140-6736(12)61728-0, https://doi.org/10.1016/S0140-6736(12)61728-0.

3. Herdy AH, López-Jimenez F, Terzic CP, et al. South American guidelines for cardiovascular disease prevention and rehabilitation. Arq Bras Cardiol. 2014;103(2 Suppl.1):1-31. ttp://dx.doi.org/10.5935/abc.2014S003

4. Guimarães FAB, Gardenghin G, Silva FMF. Reabilitação Cardíaca, tratamento e prevenção: Revisão bibliográfica. Revista Movimenta. 2015;8(1):50-9.

5. Martin BJ, Hauer $\mathrm{T}$, Arena R, et al. Cardiac rehabilitation attendance and outcomes in coronary artery disease patients. Circulation 2012;126:677-87. doi: 10.1161/CIRCULATIONAHA.111.066738, https://doi.org/10.1161/CIRCULATIONAHA.111.066738.

6. Mair V, Breda AP, Nunes MEB, Matos LD, et al. Avaliação da aderência ao programa de reabilitação cardíaca em um hospital particular geral. Einstein. 2013;11(3):278-84. http://dx.doi.org/10.1590/S1679-45082013000300004.

7. Varnfield $M$, Karunanithi $M$, Lee $C K$, et al. Smartphone-based home care model improved use of cardiac rehabilitation in postmyocardial infarction patients: results from a randomised controlled trial. Heart. 2014;100:1770-9. doi: 10.1136/heartjnl-2014-305783, https://doi.org/10.1136/heartjnl-2014-305783.

8. Braga HO, Gonzales Al, Sties SW, et al. The brasilian samba protocol for cardiac rehabilitation. Ver Bras Med Esporte. Set/Out 2015;21(5)395-9. http://dx.doi.org/10.1590/1517$\underline{869220152105136354}$

9. Kaltsatou ACH, Kouidi El, Anifanti MA, et al. Functional and psychosocial effects of either a traditional dancing or a formal exercising training program in patients with chronic heart failure: a comparative randomized controlled study. ClinRehabil. 2014;28(2):128-38. doi: 10.1177/0269215513492988, https://doi.org/10.1177/0269215513492988.

10. American College of Sports Medicine. Diretrizes do ACSM para o teste de esforço e sua prescrição [Traduzido por Giuseppe Taranto]. 7ạ ed. Rio de Janeiro: Guanabara Koogan; 2007. p. 23 
11. Malachias MVB, Souza WKSB, Plavnik FL, Rodrigues CIS, Brandão AA, Neves MFT, et al. 7ạ Diretriz Brasileira de Hipertensão Arterial. Arq Bras Cardiol 2016; 107(3Supl.3):1-83.

12. Associação Brasileira para o Estudo da Obesidade e da Síndrome Metabólica Diretrizes brasileiras de obesidade 2016 / ABESO - Associação Brasileira para o Estudo da Obesidade e da Síndrome Metabólica. - 4.ed. - São Paulo, SP. Disponível em: https://goo.gl/khYCDs.

13. Ministério da Saúde. Fundação Nacional de Saúde. Disponível em http://datasus.fns.gov.br.

14. Moraes KD, Araújo AP, Santos AF, Barbosa JMA, Martins MLB. Correlação entre o índice de massa corporal e indicadores antropométricos de risco cardiovascular em mulheres. Rev Pesq Saúde. 2015. 16(3):175-81.

15. Rolande DM, Fantini JP, Cardinalli Neto A, et al. Prognostic determinants of patients with chronic systolic heart failure secondary to systemic arterial hypertension. Arq Bras Cardiol. 2012;98(1)76-84. http://dx.doi.org/10.1590/S0066-782X2011005000123. 